\title{
Comment on: Schmidt MI, Duncan BB, Vigo A et al. (2006) Leptin and incident type 2 diabetes: risk or protection? Diabetologia 49:2086-2096
}

\author{
J. R. S. Arch
}

Received: 29 September 2006 / Accepted: 6 October 2006 / Published online: 10 November 2006

(C) Springer-Verlag 2006

To the Editor:

The report of Schmidt et al. [1] showing that high plasma leptin predicts a reduced risk of developing type 2 diabetes after adjusting the leptin level for obesity indices and fasting insulin goes a long way to rationalising a perceived disparity between studies, some of which show an association between leptin levels and increased risk of diabetes in humans, and others of which show beneficial effects of leptin injections in animal models of diabetes (references in [1]). That this disparity is merely perceived is apparent from the fact that insulin resistance or diabetes in obese animals is, as in humans, associated with high plasma leptin levels [2]. The one notable exception to this relationship is the $L e p^{o b} / L_{e p}{ }^{o b}$ mouse, which becomes obese because it does not produce functional leptin.

\section{High plasma leptin as an indicator of leptin resistance}

Schmidt et al. rightly recognise that their finding, namely that leptin levels adjusted for obesity indices and fasting insulin (as well as age, sex, study centre and ethnicity) predict reduced risk of diabetes, is consistent with the many beneficial metabolic effects of leptin described in rodents (references in [1]). However, their interpretation of the association of leptin levels adjusted only for age, sex, study centre and ethnicity with increased risk of developing diabetes in terms of leptin resistance is open to question. Like many others, they argue that the high plasma leptin

J. R. S. Arch $(\square)$

Clore Laboratory, University of Buckingham,

Buckingham MK18 1EG, UK

e-mail: jon.arch@buckingham.ac.uk levels associated with obesity probably reflect leptin resistance. In the absence of any other information, this is not a logical conclusion: leptin is produced by adipocytes, and so obesity may be the cause of the high leptin levels, rather than a consequence of leptin resistance. The only way to conclude that obesity is a consequence of leptin resistance simply from its association with high leptin levels is by defining leptin resistance as a weak ability of leptin to prevent weight gain in response to any pressure, whether environmental or genetic. This simply means that body weight is poorly controlled in the direction of weight gain, as we all know. Such a definition would not demand that lean and obese subjects differ fundamentally in their sensitivity to leptin, although the obese might be less affected by an increase in plasma leptin because they are nearer to plateau of the concentration-response curve.

\section{High plasma leptin as a cause of leptin resistance}

Nevertheless, there is other evidence showing that leptin resistance of a more fundamental nature occurs, at least in rodents, when obesity is associated with high leptin levels. The issue is: what causes this resistance? Schmidt et al. state that being overweight, overeating or a sedentary lifestyle may lead to leptin resistance with resulting hyperleptinaemia. This seems back-to-front. Is it not more likely that being overweight, overeating or a sedentary lifestyle lead to increased fat stores and hyperleptinaemia, and it is hyperleptinaemia that causes leptin resistance? Indeed, there is strong evidence that high leptin concentrations cause leptin resistance in rodents. The lethal yellow $\left(\mathrm{A}^{\mathrm{y} / \mathrm{a}}\right)$ mouse is obese, has a high plasma leptin level and is resistant to exogenous leptin, but when this mutation is 
expressed in $L e p^{o b} / L e p^{o b}$ mice so that the resultant mouse has no leptin, it becomes even more obese and yet, like the $\mathrm{Lep}^{o b} / \mathrm{Lep}^{o b}$ mouse, highly sensitive to leptin [3]. Scarpace et al. have used a more direct approach to show that leptin can produce leptin resistance. Expression of leptin using adenovirus technology in the brain of the rat results in resistance to centrally injected leptin, even though the animals initially become thinner. As leptin resistance develops, the decreased adiposity and the weight loss are reversed [4-6]. So in this model, leptin resistance is a consequence of obesity before it makes any contribution to its cause. Elsewhere [7] I have called leptin resistance elicited by high leptin levels 'secondary leptin resistance' to distinguish it from leptin resistance programmed by genes or the perinatal environment. Schmidt et al. might have supported their version of events by citing a recent report that C-reactive protein causes leptin resistance [8]. The authors of that report demonstrate, however, that leptin stimulates expression of C-reactive protein in hepatocytes, so it would appear that obesity and hyperleptinaemia are more a cause than a consequence of elevated C-reactive protein.

\section{Leptin resistance as a cause of diabetes}

Having argued that high leptin levels reflect leptin resistance, Schmidt et al. [1] propose that dysregulation of leptin action (i.e. leptin resistance) is an important pathway by which obesity causes diabetes. If so, why does the $L e p^{o b} / L e p^{o b}$ mouse, which is highly sensitive to leptin, display severely impaired glucose tolerance and insulin resistance, and, on a suitable genetic background, overt diabetes [9]? Is not another interpretation of the data by Schmidt et al., that the more leptin an individual produces for a given level of risk factors for diabetes, the more protected that individual will be from diabetes? Leptin and hence leptin resistance are associated with risk of diabetes because they are associated with risk factors for diabetes, especially obesity; they are not causes of diabetes.

Other workers have recently reported a negative association between plasma leptin and coronary heart disease in type 2 diabetes; this association was apparent only after controlling for the confounding influence of subcutaneous fat [10]. So leptin also appears to have a beneficial effect on coronary heart disease in type 2 diabetes.

\section{False analogy between insulin resistance and leptin resistance}

For diabetologists and others, it is too easy to draw analogies between insulin resistance and leptin resistance. The assumption is that since high fasting insulin levels are an indicator of insulin resistance, and insulin resistance is (with beta cell dysfunction) a prime cause of type 2 diabetes, then high leptin levels must be an indicator of leptin resistance, and leptin resistance a prime cause of obesity. There are key differences, however. Insulin resistance is primarily due to obesity (interacting with a genetic predisposition); high plasma insulin is a secondary factor. Leptin resistance, by contrast, is (at least in rodents) a major consequence of high plasma leptin. Leptin resistance is more a consequence than a cause of obesity; insulin resistance is more a cause than a consequence of diabetes. As so often in biology, the interpretation of correlations in terms of cause and effect is far from straightforward.

\section{References}

1. Schmidt MI, Duncan BB, Vigo A et al (2006) Leptin and incident type 2 diabetes: risk or protection? Diabetologia 49:2086-2096

2. Maffei M, Halaas J, Ravussin E et al (1995) Leptin levels in human and rodent: measurement of plasma leptin and ob RNA in obese and weight-reduced subjects. Nat Med 1:1155-1161

3. Boston BA, Blaydon KM, Varnerin J, Cone RD (1997) Independent and additive effects of central POMC and leptin pathways on murine obesity. Science 278:1641-1644

4. Scarpace PJ, Matheny M, Zolotukhin S, Tumer N, Zhang Y (2003) Leptin-induced leptin resistant rats exhibit enhanced responses to the melanocortin agonist MT II. Neuropharmacology 45:211-219

5. Scarpace PJ, Matheny M, Tumer N, Cheng KY, Zhang Y (2005) Leptin resistance exacerbates diet-induced obesity and is associated with diminished maximal leptin signalling capacity in rats. Diabetologia 48:1075-1083

6. Zhang Y, Scarpace PJ (2006) The role of leptin in leptin resistance and obesity. Physiol Behav 88:249-256

7. Arch JR (2005) Central regulation of energy balance: inputs, outputs and leptin resistance. Proc Nutr Soc 64:39-46

8. Chen K, Li F, Li J et al (2006) Induction of leptin resistance through direct interaction of C-reactive protein with leptin. Nat Med 12:425-432

9. Coleman DL, Hummel KP (1973) The influence of genetic background on the expression of the obese $(O b)$ gene in the mouse. Diabetologia 9:287-293

10. Ruige JB, Mertens I, Considine RV, Paelinck BP, Van Gaal LF (2006) Opposite effects of insulin-like molecules and leptin in coronary heart disease of type 2 diabetes Preliminary data. Int $\mathrm{J}$ Cardiol 111:19-25 